\title{
Intermédialités
}

Histoire et théorie des arts, des lettres et des techniques

Intermediality

History and Theory of the Arts, Literature and Technologies

\section{Interartialité et remédiation scénique de la peinture}

Tatiana Burtin

Numéro 12, automne 2008

Mettre en scène

Directing

URI : https://id.erudit.org/iderudit/039232ar

DOI : https://doi.org/10.7202/039232ar

Aller au sommaire du numéro

Éditeur(s)

Centre de recherche sur l'intermédialité

ISSN

1705-8546 (imprimé)

1920-3136 (numérique)

Découvrir la revue

Citer cet article

Burtin, T. (2008). Interartialité et remédiation scénique de la peinture.

Intermédialités / Intermediality, (12), 67-93. https://doi.org/10.7202/039232ar
Résumé de l'article

Le propos de cet article est d'approfondir la notion d'interartialité, dont l'archéologie a été élaborée par Walter Moser, et d'étendre son application à des cas peu fréquents afin de la mettre à l'épreuve. La théâtralisation de la peinture sur la scène, examinée dans deux spectacles contemporains, «Art » de Yasmina Reza et Seuls de Wajdi Mouawad, offre un bon exemple de résistance d'un art à un autre. La mise en scène d'un tableau interroge le statut et le sens de l'oeuvre d'art hors du musée, mais aussi la spécificité de l'esthétique théâtrale, et sa capacité à jouer avec la transparence et l'opacité des arts et des médias qu'elle accueille dans son espace. Par la présence du tableau (" réelle " ou reproduite par image vidéo) sur la scène, et par la place des autres corps, notamment celui de l'acteur, face à elle, l'art de la peinture permet au spectateur d'appréhender une nouvelle profondeur de la scène, quelque peu oubliée de nos jours par la perception bidimensionnelle des arts de l'image, appréhension qui remet en cause l'esthétique théâtrale de la distance et de l'illusion. 


\title{
Interartialité et remédiation scénique de la peinture
}

\author{
TAtiana BURTIN
}

e théâtre a souvent été défini comme un «hypermédia», à la fois trans- parent et opaque, qui intègre les autres arts, médias et nouveaux médias sans toucher à leur spécificité, ou à leur «essence»: «The first principle is that theatre is a hypermedium that incorporates all arts and media and is the stage of intermediality ${ }^{1}$.» Chiel Kattenbelt prend appui sur les travaux de Jan Mukařovskýz, pour qui les arts individuels perdent leur autonomie sur scène pour devenir un nouvel art: «the theatre performance is a contexture (a weaving of strands together to create a texture) as opposed to a composition of individual elements ${ }^{3} »$. L'essence de la performance théâtrale, phénomène transitoire, est la «théâtralisation» (theatricalization) qui émerge des relations sans cesse changeantes entre ses composants spatiaux et temporels. Le théâtre est donc plus qu'un hypermédia, c'est le seul art capable d'incorporer les autres sans être dépendant d'aucun d'entre eux pour être du théâtre ${ }^{4}$. L'hypermédialité du théâtre expliquerait ainsi sa nature interartielle. Mais cette interartialité semble connaître au moins deux niveaux qui compliquent d'emblée une claire définition du phénomène.

1. Freda Chapple, «Key Issues in Intermediality in Theatre and Performance», dans Freda Chapple et Chiel Kattenbelt (dir.), Intermediality in Theatre and Performance, Amsterdam et New York, Rodopi, 2006, p. 20.

2. Publiés en recueil dans Jan Mukařovský, Structures, Sign and Function: Selected Essays, trad. John Burbank et Peter Steiner, New Haven, Yale University Press, 1978.

3. Chiel Kattenbelt, «Theatre as the Art of the Performer and the Stage of Intermediality", dans Freda Chapple et Chiel Kattenbelt (dir.), Intermediality in Theatre and Performance, p. 31 .

4. Chiel Kattenbelt, «Theatre as the Art of the Performer and the Stage of Intermediality", p. 32. 
La scénographie a bien intégré dans son discours les techniques de la peinture, de la sculpture, de la musique, comme moyens destinés à créer une illusion théâtrale; de même, les principes de l'architecture sont importants pour le montage d'un plateau, par rapport à l'œil du spectateur. Ces diverses techniques - il s'agit ici de "techniques", ou encore de "médias», et non pas d'«arts» - s'imbriquent afin de réaliser la «texture» du théâtre. Il s'agirait là d'une première forme de «théâtralisation » d'un art, c'est-à-dire d'inclusion dans un discours artistique de techniques et de trouvailles importées d'autres arts. En résumé, c'est ici le théâtre comme "scène de l'intermédialité", ou au sens strict d'hypermédia (qui intègre divers médias), qui est considéré. Par ailleurs, nombre de spectacles contemporains illustrent la capacité de la scène à accueillir d'autres expressions artistiques, en mêlant au jeu théâtral danse, mime, cirque, vidéo, cinéma, etc., qui sont dès lors théâtralisés, bien qu’encore reconnaissables par leurs composantes propres (pas de danse fixes ou mouvements rythmiques, accessoires ou personnages de cirque, écrans, caméras, etc.). Je considère cette transposition - il est difficile de trouver un terme exact - d'un art au théâtre comme le second niveau de «théâtralisation » d'un art, au sens d'intégration dans l'espace et le discours théâtraux d'une expression artistique pleine et forte, qui impose sur la scène son propre discours, voire sa propre esthétique (mais cela reste à prouver), ainsi que la majeure partie de sa médialité, et non plus seulement quelques aspects.

Force est de constater que les arts qui, de cette dernière manière, se marient le mieux à l'expression scénique, sont la plupart du temps «vivants». Les arts plastiques semblent quant à eux résister à la «théâtralisation » qu'impose la scène à tout art et à tout média5. Ce n'est pas le cas d'autres arts fondés sur le mouvement, comme le cinéma, qui a donné un grand nombre de films sur la peinture par exemple, à travers une véritable relation interartielle, comme l'a démontré Walter Moser dans un article récent ${ }^{6}$. Il y distingue en effet deux types de relations entre les arts à partir de la traditionnelle comparaison entre

5. Je ne parle pas ici du débat sur la théâtralité de la peinture, développé dans les travaux du critique et historien de l'art Michael Fried (Michael Fried, Contre la théâtralité: du minimalisme à la photographie contemporaine, trad. Fabienne Durand-Bogaert, Paris, Gallimard, coll. «NRF Essais», 2007), mais de l'intégration de la peinture et de sa médialité sur la scène de théâtre.

6. Walter Moser, «L'interartialité: pour une archéologie de l'intermédialité», dans Marion Froger et Jürgen E. Müller (dir.), Intermédialité et socialité: histoire et géographie d'un concept, Münster, Nodus Publikationen, coll. «Film und Medien in der Diskussion », vol. 14, 2007, p. 69-92. 
poésie et peinture: une «relation d'égalité-réciprocité» basée sur une esthétique commune (l'imitation), qui permettrait un dialogue «libre» entre les arts, qu'il évoque mais dont il ne donne pas d'exemple; une « relation de domination » d'un art sur l'autre, ou encore de remédiation d'un art par l'autre, qu'il semble considérer comme la seule valide théoriquement ${ }^{7}$. S'il s'applique à retracer la «tradition de l'interartialité ${ }^{8}$ » depuis l'Ut pictura poesis d'Horace, il cherche avant tout à en élaborer une nouvelle définition par la notion d'intermédialité. En effet, la relation entre arts ne se conçoit pas sans relation aux médias: «si l'interartialité implique toujours de l'intermédialité, cette proposition ne saurait cependant être inversée ${ }^{9} »$.

Le problème de l'interartialité comme remédiation d'un art par un autre, que Walter Moser distingue apparemment de la «relation art-média ${ }^{10}$ ", ainsi que l'ambiguïté du concept de «théâtralisation» sont au fond des questions assez similaires. Leurs points communs apparaissent d'autant mieux quand on compare deux arts dont les effets sur le public ou l'esthétique diffèrent, comme le théâtre et la peinture ${ }^{11}$. En effet, si peinture et théâtre s'inscrivent tous deux dans des «cadres» (pictural - encadrement - , et scénique - rideau, rampe, etc.), la peinture, notamment moderne, est un art de la "présenteté », selon la terminologie de Michael Fried ${ }^{12}$, alors que le théâtre est un art de la présence, qui entraînent chacun des effets particuliers sur le spectateur ${ }^{13}$. Il semble que ce soit ce point qui rende difficile la médiation de la peinture par le théâtre. Comment dès lors théâtraliser, pour reprendre l'expression de Mukařovský, l'œuvre produite

7. Walter Moser, «L’interartialité: pour une archéologie de l'intermédialité», p. 70-71.

8. Walter Moser, «L'interartialité: pour une archéologie de l'intermédialité », p. 70.

9. Walter Moser, «Puissance baroque dans les nouveaux médias. À propos de Prospero's Books de Peter Greenaway ", Cinémas, vol. 10, n²-3, "Cinéma et intermédialité », sous la direction de Silvestra Mariniello, printemps 2000, p. 45.

10. Walter Moser, «L'interartialité: pour une archéologie de l'intermédialité», p. 90-91. Moser résume ainsi ses conclusions sur les deux cinéastes qu'il cite dans sa démonstration: «Chez Jarman, [...] [l’]interartialité est utilisée pour capter la médialité de la peinture. Chez Godard, en revanche, [...] cette interartialité (ou plutôt cette relation art-média) est utilisée pour capter la médialité du film.»

11. Bien que le théâtre, art du «tableau», suscite souvent la comparaison avec la peinture, comme le fait Diderot, mais pour s'en démarquer fortement.

12. Michael Fried, Contre la théâtralité: du minimalisme à la photographie contemporaine, p. 139 .

13. Chiel Kattenbelt, «Theatre as the Art of the Performer and the Stage of Intermediality », p. 32 . 
par l'art de peindre? Explorons le cas de deux pièces du répertoire contemporain où la peinture, élément central de l'intrigue, apporte une véritable réflexion sur l'art et l'esthétique, autant picturale que théâtrale.

"Art», de Yasmina Reza, a été montée pour la première fois le 28 octobre 1994 à Paris à la Comédie des Champs-Élysées ${ }^{14}$ avec Pierre Vaneck, Fabrice Luchini et Pierre Arditi dans les rôles respectifs de Marc, Serge et Yvan ${ }^{15}$. Seuls, de Wajdi Mouawad a été créée à l'Espace Malraux, scène nationale de Chambéry et de la Savoie, le 4 mars 2008, puis reprise en tournée en France et au Québec ${ }^{16}$. J'ai choisi ces deux pièces, très différentes, voire inégales, dans leur inspiration, leur esthétique, leur réalisation et leur cible, car elles intègrent physiquement dans leur scénographie, et surtout dans leur fable, des tableaux ${ }^{17}$, connus ou non, hors de leur «cadre» naturel, non seulement hors de leur encadrement, mais aussi de leur environnement habituel, le musée, et de sa "mise en scène », la muséographie ${ }^{18}$. Ce faisant, elles interrogent le statut et le sens de l'œuvre d'art une fois extirpée de son institution, et, en retour, la spécificité de l'esthétique théâtrale, et la capacité de la scène à intégrer et à jouer avec la transparence et l'opacité des arts et des médias qu'elle accueille dans son espace.

14. Comédie mise en scène par Patrice Kerbrat; décor: Edouard Lang; lumières: Laurent Béal. C'est une comédie de boulevard à la fois efficace et branchée, sur le thème de l'art et de l'amitié.

15. Voir Yasmina Reza, Théâtre. L'homme du hasard. Conversations après un enterrement. La traversée de l'hiver. "Art», Paris, Albin Michel, coll. "Le Livre de Poche», 1998.

16. Dont un mois à Montréal, au Théâtre d'Aujourd'hui, du 9 septembre au 9 octobre 2008. Texte, mise en scène et jeu: Wajdi Mouawad; scénographie: Emmanuel Clolus; éclairage: Éric Champoux; réalisation sonore: Michel Maurer; réalisation vidéo: Dominique Daviet.

17. Ce terme n'est pas à prendre au sens moderne de découpage scénique, ni de suspens dans l'action, comme dans le drame bourgeois, ni même d'élément purement décoratif, bien qu'il appartienne au décor, mais sémantiquement, comme un protagoniste à part entière des pièces étudiées.

18. À ma connaissance, les exemples de ce genre n'abondent pas au théâtre. L'adaptation de Maîtres anciens de Thomas Bernhardt par Denis Marleau (1995) accroche virtuellement le tableau sur le quatrième mur; les performances de «peintures sur scène » de Robert Lepage n’ont pas de fable au sens classique du terme. 


\section{I- ENJEu du déPLACEMENT De la RELATION INTERARTIELle Au thÉÂtre}

Dans son article sur l'interartialité considérée, entre autres, comme une «archéologie de l'intermédialité », Walter Moser explore les relations entre deux arts, qui sont, par définition, des médias:

La base de mon argument est, succinctement, la suivante: la relation entre les arts, par implication, comporte toujours aussi des enjeux intermédiatiques, même si ceux-ci ne sont pas explicités en tant que tels, étant donné que tout art inclut la «médialité». L'inversion de cette affirmation n’est pas nécessairement vraie: l'interaction entre les médias peut s'articuler sans que les arts y soient impliqués. Toujours porté par un soubassement intermédial, l'art se distingue pourtant du média par des déterminations - par exemple de nature esthétique - qui lui assignent un champ plus étroit ${ }^{19}$.

Si Moser ne traite jamais directement de ces différences d'ordre esthétique dans ce texte, il y explique que la médialité d'un art tend à s'effacer dans un semblant de transparence dans toute production artistique, mais devient nécessairement apparente quand deux arts entrent en interaction : l'opacité du média émerge en tant qu'objet de connaissance d'une relation intermédiale ${ }^{20}$. Ces relations révèlent peut-être l'opacité et la capacité de représentation et de remédiation de tout média, mais il me semble qu'un authentique questionnement sur l'interartialité devrait aborder davantage les notions d'art et d'esthétique elles-mêmes. Je ne considérerai donc pas une définition purement médiale de ce dernier terme, celle de passage de techniques, de matériaux, d'économies, de socialités ou d'institutionnalités propres à certains arts, à l'hypermédia théâtre. Bien que la réflexion intermédiale de Moser soit indispensable à tout propos sur l'interartialité, je parlerai, dans le cas du théâtre, d'art non seulement hypermédial mais également «hyperesthétique », c’est-à-dire capable d'incorporer les problèmes de perception liés à un autre art, voire de faire connaître à son spectateur des expériences esthétiques plus nombreuses grâce

19. Walter Moser, «L'interartialité: pour une archéologie de l’intermédialité », p. 69 (nous soulignons).

20. «Ce serait l'intermédialité implicite de cette interartialité qui obligerait la littérature à révéler sa propre médialité. Je dis "obligerait" car, selon une certaine loi des médias, la médialité d'un art, qui tend à s'effacer dans un semblant de transparence, devient nécessairement apparente quand deux médias différents entrent en jeu et interfèrent [...]. Le média apparaît alors, prenant la consistance d'une opacité. Il émerge donc, en tant qu'objet de connaissance d'une relation intermédiale qui l'aurait (toujours déjà) précédé.» Walter Moser, «L'interartialité: pour une archéologie de l'intermédialité», p. 80. 
au jeu de l'interartialité (par exemple, le point de vue de l'artiste sur son œuvre en train d'être créée).

Le premier film commenté par Moser, Caravaggio (1986) de Derek Jarman, narrativise la fabrication d'un tableau à travers plusieurs techniques que nous verrons plus loin. Moser ne relève pas d'allusions dans le film aux goûts du peintre ni à la conception qu'il a de son art. Il renonce sciemment à développer la narrativisation des «moments fertiles », qu'il évoque pourtant dans la description des «tours de passe » du cinéaste, «procédés esthétiques de la conversion de la peinture en film ${ }^{21} »$ :

Il est vrai que beaucoup des films abordent la peinture par le biais narratif et dramatique d'une biographie de peintre, ce qui constitue en soi un phénomène de narrativisation intéressant. Néanmoins je m’intéresse ici davantage aux moments de corps à corps entre film et peinture auxquels le récit biographique sert souvent de cadre et parfois de prétexte pour sa mise en film².

La problématique de la narrativisation biographique des tableaux appartient aux marges du modèle interartiel tel que le conçoit Lessing ${ }^{23}$. Sans reprendre à son compte ce schéma trop étroit de classification des arts, Moser s'appuie sur ses bases théoriques et sur sa prise en compte des cas litigieux pour démontrer la médialité de tout $\mathrm{art}^{24}$. Il cite les modèles du Caravage et la violence des relations que le peintre entretient avec eux, mais il ne revient sur l'aspect narratif de ces scènes qu'en note ${ }^{25}$ : "Que Jarman reproduise, par son interprétation de la biographie de Caravaggio, le topos de l'artiste maudit est moins important pour mon propos ici que le fait que l'interaction avec ses modèles, indispensables pour

21. Walter Moser, «L'interartialité: pour une archéologie de l'intermédialité », p. 83. 22. Walter Moser, «L'interartialité: pour une archéologie de l'intermédialité», p. 82.

23. Lessing, dans son ouvrage intitulé Laokoon oder Über die Grenzen der Malerei und Poesie, publié en 1766, part de la traditionnelle relation entre poésie et peinture («ut pictura poesis» d'Horace) pour remédier à la confusion entre les arts, qu'il considère comme une marque de «mauvais goût». S'il existe une communauté d' «esthétique » et d'effet à produire entre peinture et poésie, ces arts divergent sur le plan de leur «matérialité et [de] leurs médias qui déterminent des modalités de représentation ». Walter Moser, «L'interartialité: pour une archéologie de l’intermédialité», p. 72.

24. Walter Moser, «L'interartialité: pour une archéologie de l'intermédialité», p. $70-73$.

25. Notes 43 et 44. Caravaggio est blessé par Ranuccio au flanc droit, ce qui permettra de substituer son visage à celui du Christ dans la scène avec saint Thomas. De même, le cadavre de Léna, tuée par Ranuccio, deviendra dans le film le modèle pour le tableau La mort de la Vierge. 
son travail de peintre, le situe dans un milieu social très spécifique ${ }^{26}$ ", précise-t-il. Il s'attache donc à démontrer l'insistance sur la médialité d'un art (dans ce cas précis de la socialité et de la «technicalité » de la peinture, à travers la façon dont le peintre choisit ses modèles, les paie, les fait poser, etc.) que donne à voir un autre média, dont le statut artistique a d'ailleurs longtemps été discuté, comme il le souligne lui-même 27 . Ainsi, c'est moins le processus de l'interartialité, considéré comme une confrontation de moyens de réalisation et une influence réciproque, voire une interpénétration d'esthétiques différentes, que la révélation de la médialité d'un art placé sous le regard d'un autre, que cet exemple veut mettre en lumière.

Le deuxième exemple qu'analyse Moser, Passion (1982) de Godard, traite de la difficulté à créer un film sur la peinture, à faire dialoguer les arts dans un média nouveau, complexe, dont les possibilités sont dévoilées par la pratique cinématographique du réalisateur. Dans ce cas-ci, la question de la narrativité est développée de façon conséquente ${ }^{28}$, ainsi que l'esthétique du film en relation avec celle de la peinture, à travers des «méta-discussions sur la fabrication du film, son public cible ou potentiel et ses qualités esthétiques ${ }^{29}$ ». Godard re-médie, au sens qu'ont donné au terme Bolter et Grusin, l’art pictural par le septième art. La peinture de Rembrandt, par exemple, inspire le style du cinéaste Jerzy filmant la reconstitution de La ronde de nuit, dont il copie les contrastes lumineux ${ }^{30}$ : «l'ancien média participe à la configuration du nouveau ${ }^{31} »$. De même, les séquences se succèdent dans un désordre qui évoque un «chaos créateur dont aucun "produit fini" ne résulte», et que Moser associe, «étonnamment», à «l'esthétique romantique du fragment en tant que work in progress ». Moser pose des jalons importants pour la réflexion interartielle, mais,

26. Walter Moser, «L'interartialité: pour une archéologie de l'intermédialité », p. 86.

27. «Si le statut médiatique du cinéma ne semble pas faire problème, ni l'identification du film comme produit de l'industrie culturelle, sa reconnaissance en tant qu'art n'alla pas de soi. Est-ce chose acquise? Un indice positif pourrait provenir du fait que deux des plus récentes publications sur la question furent écrites par deux historiennes de l'art [...]. » Walter Moser, «L'interartialité : pour une archéologie de l'intermédialité », p. 81.

28. Walter Moser, «L'interartialité: pour une archéologie de l'intermédialité», p. $87-88$.

29. Walter Moser, «L'interartialité: pour une archéologie de l'intermédialité», p. 90.

30. Dans ce passage, la voix hors-champ fait aussi un commentaire sur «l'utilisation de la surface du tableau transposable à l'écran ». Walter Moser, «L'interartialité: pour une archéologie de l'intermédialité », p. 88, note 51.

31. Walter Moser, «L'interartialité: pour une archéologie de l’intermédialité», p. 88. 
fidèle à sa démonstration, il ne retient que les procédés qui mettent en valeur, encore une fois, la médialité, non plus de la peinture comme chez Jarman, mais du cinéma. Il semble que ce soit l'incapacité même du film de Jerzy à parler peinture qui incite Moser à voir une simple « relation art-média » à l'œuvre dans Passion plutôt qu'une relation pleinement interartielle. Comme nous l'avons déjà remarqué, pour lui, Godard «utilise la relation interartielle entre film et peinture pour faire apparaître la médialité complexe du film² ${ }^{32}$. En ne filmant qu’ « exceptionnellement» le résultat des reconstitutions du cinéaste, qui sont interrompues par des «difficultés d'ordre matériel, technique, personnel, financier et conceptuel » propres à toute production cinématographique, Godard fait voir et raconte au spectateur le média «cinéma » et non le média «peinture». Un procédé de narrativisation de la peinture aurait certainement consisté à filmer les versions achevées des tableaux, comme prêtes à être transposées sur une toile. Si la peinture, "ancêtre du cinéma en tant qu'art et média de l'image », peut inspirer un film, il n'est en revanche pas le même objet, qui se définit sous nos yeux, dans le film de Godard, par l'affirmation de sa médialité propre; aussi le film échoue-t-il in fine à «reproduire» les tableaux. Il ne peut copier l'art des musées, car le cinéma a développé une autre esthétique que l'imitation picturale, mais toujours grâce à l'image. Cette étape correspond à la mutation théorique de l'interartialité au $19^{\mathrm{e}}$ siècle, au moment où « le modèle artistique n'est plus un art qui imite et qui copie, mais un art non représentatif dont on rend compte non pas en termes de vérité et d'adéquation à un objet à représenter, mais en termes d'énergie émotive ${ }^{33} »$.

Si le «média cinéma » résiste à l'intégration totale d'un art à la fois concurrent et inspirant, allant jusqu'à faire échouer un projet de film qui prétend s'ériger au rang d'œuvre d'art par ses «qualités esthétiques », comment poursuivre le dialogue interartiel? Passion nous fait apercevoir une réponse: c'est un film réussi, achevé, sur un film inachevé qui échoue, inachevé. Le film qui achoppe sur la médialité de la peinture, qu'il cherche vainement à s'approprier mais dont il rend compte malgré tout dans son élaboration même, est mis en abyme dans un film qui met en scène son histoire et sa médialité. Par l'achèvement du processus autoréflexif sur sa propre esthétique, réalisé dans un long-métrage qui en démonte tous les rouages, le film de Godard a triomphé de la limite imposée par sa médialité. Je dirais que, au lieu que ce soit le film qui « utilise la rela-

32. Walter Moser, «L'interartialité: pour une archéologie de l'intermédialité», p. 88. 33. Walter Moser, «L'interartialité: pour une archéologie de l'intermédialité », p. 74. 
tion interartielle» pour «donn[er] à voir et à connaître la médialité de l'art ${ }^{34}$ », ce sont les rapports interartiels au sein même d'une œuvre d'art qui façonnent le média. La nuance est fine et semble malaisée à expliquer. Il ne s'agit pas de récuser le «soubassement médial"35" de tout art; mais je fais l'hypothèse que c'est l'esthétique colportée par un art qui apparaît dans le dialogue interartiel et intermédial dans toute sa spécificité, au-delà de sa médialité. Autrement dit, plus qu'un rapport symétrique entre deux arts, dont l'un cherche (à imposer) sa médialité grâce à l'autre, je considère ici l'interartialité comme un rapport dialectique entre deux esthétiques qui n’évacuerait pas cependant la dimension médiatique. Il se pourrait aussi que les esthétiques soient elles-mêmes des éléments de médialité.

Le rapport peinture-théâtre semble un angle intéressant pour éclairer d'un jour nouveau ce problème, car il est, comme le cinéma, un cas de «transgression constitutive, puisqu'il a intégré dans un même média les deux principes qu'a séparés Lessing ${ }^{36}$ », textuellement, dans son modèle de distinction entre l'objet poésie et l'objet peinture, à savoir «déroulement d'une action » dans le temps et «corps dans l'espace ${ }^{37}$ ». On peut en effet considérer le théâtre comme un art de l’image, qui présenterait au spectateur une série de «tableaux» mobiles $^{38}$. Par

34. Walter Moser, «L'interartialité: pour une archéologie de l'intermédialité», p. 91. 35. Walter Moser, «L'interartialité: pour une archéologie de l'intermédialité», p. 76. 36. Walter Moser, «L'interartialité: pour une archéologie de l'intermédialité», p. 83.

37. Walter Moser, «L'interartialité : pour une archéologie de l’intermédialité », p. 72. Cela n'est évidemment plus valable pour la peinture moderniste, où tout corps et même toute forme ont disparu de la surface de la toile. Voir Clement Greenberg, «Towards a Newer Laocoon ", 1940, dans John O'Brian (dir.), The Collected Essays and Criticism: Perceptions and Judgments, 1939-1944, vol. 1, Chicago, University of Chicago Press, 1986. Je reparlerai de ce débat à propos d'«Art».

38. Conception surtout revalorisée par le théâtre contemporain, notamment par Brecht, qui procède à un découpage en «tableaux » de ses pièces, en fonction des changements d'espaces ou d'espace-temps. Fernand Léger, dans Fonctions de la peinture, recueil d'articles sur sa théorie de l'art, conçoit aussi le cinéma comme «des images mobiles présentées comme un tableau» (Fernand Léger, «Essai critique sur la valeur du film d'Abel Gance, La roue », Fonctions de la peinture, Paris, Gallimard, coll. «Folio Essais», 1997, p. 162). Le but du cinéma, l’image mobile, se rapproche de la peinture grâce aux possibilités techniques de la caméra; il laisse au théâtre l'histoire et le scénario. Robert Bresson considère, quant à lui, le cinéma ou «théâtre photographié » comme l'équivalent de la reproduction photographique d'une toile de peintre ou d'une sculpture; elle perd la valeur et le prix de l'original (Robert Bresson, Notes sur le cinématographe, Paris, Gallimard, coll. «Folio», 1995, p. 18-19). 
son «mécanisme technique ", basé sur le déploiement dans l'espace du corps de l'acteur, il fait percevoir le déroulement dans le temps d'une action, à travers une histoire, de même que la séquence des images fixes au cinéma crée la durée. En revanche, il ne peut évacuer l'élément temps pour faire apparaître, comme le cinéma, la médialité de la peinture comme Jarman le peut par la caméra, en montrant sa matérialité, son économie, sa technicalité, sa socialité, son institutionnalitée ${ }^{9}$. À la rigueur, il peut faire ressortir sa propre médialité. Béatrice Picon-Vallin décrit de tels procédés de grossissement du corps de l'acteur, «matière première » du théâtre, par les écrans vidéo placés sur scène ou les techniques d'éclairage ${ }^{40}$. La focalisation de l'attention du spectateur sur le mouvement d'un petit doigt, par l'arrêt de toute autre action, dans le Révizor (1926) de Meyerhold, l'éclairage intense des acteurs, le rapprochement acteurspectateur que permet la vidéo, par le grossissement des visages des acteurs dans le Marchand de Venise (1994) de Peter Sellars, ou les gros plans sur les pieds des personnages d'un des Traités des Passions (1995-2000) de Jean-François Peyret, sont des moyens de «rendre opaque» la médialité du théâtre. L’opacité de la peinture, qui permet sa remédiation filmique en devenant un « objet de représentation » dans le média cinéma ${ }^{41}$, empêche précisément sa remédiation directe par le théâtre. Le déploiement de la matérialité de la peinture est en effet limité sur une scène: un puits de lumière sur un acteur en train de peindre, ou une captation vidéo de pigments retransmise en gros plan, pour reprendre les exemples de Moser, pourraient être des solutions. Reste la possibilité de montrer sa médialité économique (l'achat du tableau, sa transaction, son prix et sa valeur, comme dans «Art» par exemple); sa technicalité (préparation des pigments, mais la distance du spectateur est un problème; pose du modèle, application de la peinture, etc.); sa socialité (fréquentation des modèles, mais aussi visiteurs dans un musée ${ }^{42}$, amis

39. Walter Moser, "L’interartialité: pour une archéologie de l'intermédialité», p. $84-86$.

40. Béatrice Picon-Vallin, «Hybridation spatiale, registres de présence», dans Béatrice Picon-Vallin (dir.), Les écrans sur la scène: tentations et résistances de la scène face aux images, Paris, L'Âge d'Homme, coll. «Th XX», Série Études, 1998, p. 20.

41. Walter Moser, «L'interartialité: pour une archéologie de l'intermédialité», p. 85.

42. Comme dans Maîtres anciens de Thomas Bernhard (Thomas Bernhard, Maîtres anciens: comédie, Paris, Gallimard, coll. «Folio», 1991), roman adapté au théâtre par Denis Marleau (à ceci près que le tableau n'apparaît jamais, car situé «sur le quatrième mur »), Théâtre sans animaux ou Musée haut, musée bas de Jean-Michel Ribes. Voir JeanMichel Ribes, Théâtre sans animaux: neuf pièces facétieuses, Arles, Actes Sud-Papiers, coll. «Théâtre», 2001. La pièce a été créée à Paris, au Théâtre Tristan-Bernard, en août 
du collectionneur en visite, etc.); son institutionnalité (mécènes particuliers ou commandes publiques, collectionneurs et rats de galeries d'art, etc.). De même, la transparence médiale, l'auto-annulation du média qui mène l'art à s'affirmer ${ }^{43}$, est-elle valable dans le cas du théâtre? Comment nier sa médialité? Faudrait-il supprimer décor, scène, comédiens, en les recréant virtuellement?

«Art» et Seuls, à la différence de l'exposition Métissages de Robert Lepage par exemple ${ }^{44}$, proposent non d'effacer la médialité du théâtre, mais d'encadrer pour ainsi dire une médialité de la peinture, à savoir son cadre, dans sa propre esthétique. La pièce de Yasmina Reza présente aux yeux des spectateurs, donc à leurs interrogations, un tableau blanc, dans tout le troublant mystère de sa surface; la scène de théâtre se superpose presque à la salle de musée. L'esthétique picturale, c'est-à-dire la relation peinture-spectateur est ici transposée telle quelle sur le média théâtre pour mettre en relief les problèmes que pose cette esthétique par des moyens proprement théâtraux (intrigue, jeu des acteurs, postures mimant celles des peintres devant un tableau, mouvements du tableau-personnage de la fable, etc.). Le spectacle de Wajdi Mouawad, quant à lui, sur-remédie en quelque sorte le tableau de Rembrandt, Le retour du fils prodigue, en le projetant sur un mur écran, qui délimite un nouveau cadre pour cerner un sens particulier du tableau que Mouawad intègre dans une fable autobiographique, ou par l'interaction de son propre corps (il est seul en scène) sur et dans le tableau, qui perturbe son cadre naturel. L'esthétique théâtrale semble ici dialoguer avec l'esthétique picturale et la développer au-delà de la simple relation peinture-spectateur, jusqu'à faire connaître à ce dernier, par procuration, des expériences inédites, celle du peintre devant son tableau, et même la fusion de l'artiste au cour de son œuvre.

2001. Jean-Michel Ribes, Musée haut, musée bas, Arles-Montréal, Actes Sud-Leméac, coll. «Babel». La pièce a été créée à Paris, au théâtre du Rond-Point, en septembre 2004 .

43. Walter Moser, «L'interartialité: pour une archéologie de l'intermédialité», p. $77-80$.

44. Montée au Musée de la civilisation de Québec du 3 mai 2000 au 29 juillet 2001. La muséologie était un défi pour l'homme de théâtre: "un médium qui m’était totalement étranger. [...] Cela m'est apparu comme un tout autre langage, faisant appel à une toute [sic] autre structure narrative pour arriver à raconter une histoire ou développer un propos », dit-il dans Robert Lepage, Métissages, racontée par ses artisans, monographie d'une exposition, Québec, Musée de la civilisation, mai 2001, p. 24. 


\section{II- Problème de CADRes: l'ART des musées Au théÂtre}

Robert Lepage et Rembrandt ont en rapport le cadre comme espace de travail. L'un peint dessus, l'autre projette dessus.

\section{Wajdi Mouawad ${ }^{45}$}

Parler de peinture au théâtre, c'est interroger son cadre technique et esthétique. Plusieurs problèmes limitent l'intégration des tableaux, qui appartiennent à l'histoire de l'art et surtout au discours et à l'institution muséale, à l'espace distancié de la scène. Le tableau semble pour ainsi dire mal s'encadrer à la scène, car celle-ci ne peut devenir «transparente», comme le film le peut pour donner une impression de «corps à corps » avec la peinture, par les différentes techniques que relève Moser grâce à l'exemple de Jarman. Celui-ci «insiste par des gros plans tout particulièrement sur les pâtes, les huiles et les pigments qui se trouvent sur la table [de l'atelier du Caravage] [...] La technique cinématographique du gros plan de la main et du pinceau qui applique la peinture sur la toile a pour effet de «faire disparaître » tant l'objet représenté du tableau que le sujet de l'acte de peindre, et, en échange, de faire découvrir la matérialité picturale qui oblitère la transparence du tableau ${ }^{46}$. $\gg$ Plus loin, Moser tire une seconde conclusion de ce «corps à corps » entre peinture et film :

Jarman réussit à donner une intensité sans pareille au corps à corps dans lequel il engage peinture et film, un corps à corps qui dépasse de loin le traitement thématique ou le cadre narratif et qui va jusqu'à nous induire en erreur sur les niveaux de réalités impliqués dans le chassé-croisé entre film et peinture et entre art et média ${ }^{47}$.

En revanche, il est difficile de montrer sur scène une œuvre d'art, ou plutôt une simple copie - puisqu'il est inimaginable qu'un metteur en scène demande à un musée de sortir une œuvre de ses murs à l'occasion d'un spectacle, pour des raisons techniques et financières évidentes. On pense d'emblée aux problèmes d'éclairage, de placement, de mise en valeur sur la scène, de la distance qui la séparerait du spectateur, l'empêchant de la contempler comme il pourrait le

45. Citation tirée de ses carnets de travail présentés dans l'édition du texte, qui retrace la genèse du spectacle. Wajdi Mouawad, Seuls: chemin, texte et peintures, Montréal-Arles, Léméac-Actes Sud, coll. «Théâtre», 2008, p. 72.

46. Walter Moser, «L'interartialité: pour une archéologie de l'intermédialité », p. 85.

47. Walter Moser, «L'interartialité: pour une archéologie de l’intermédialité», p. 86 (nous soulignons). 
faire dans un musée, ou, à la rigueur, devant un film qui présenterait ses détails. Dans l'espace théâtral, l'œuvre descend de son piédestal pour se placer sous les feux de la rampe, domaine de l'acteur. Elle n'est plus le centre des regards, ne peut plus susciter l'admiration et l'émotion possibles par une approche directe, subjective, car elle est recadrée, redéfinie par un espace et une institution, donc un discours esthétique ${ }^{48}$ - Walter Moser parle de «sémantisme du média », c'està-dire des moyens, en lien avec sa matérialité, qui lui sont propres pour retranscrire des expériences, du sens ${ }^{49}$ - tout aussi pesants que ceux du musée, mais qui ne sont plus siens. Surtout, l'œuvre ainsi transposée n'instaure plus le même rapport esthétique, au sens premier de perception, entre elle et le spectateur. Les pièces que nous avons choisi de commenter apportent deux types intéressants de solution, qui nouent un rapport original au spectateur : "Art» offre une critique de l'esthétique muséale en la transposant directement sur la scène et dans la fable, tandis que Seuls caractérise l'esthétique théâtrale grâce à la présence d'un autre art, donc d'un autre média. Disons, pour donner un point de comparaison, qu' «Art» correspond, dans la démarche analysée par Moser, à Caravaggio de Jarman, alors que Seuls correspondrait sous ce rapport à Passion de Godard.

Dans «Art», le décor, «le plus dépouillé, le plus neutre possible ${ }^{50}$ », évoque les appartements de Marc, Serge et Yvan, trois amis de longue date. La disposition du décor ne change pas, «sauf l'œuvre de peinture exposée». Dans la captation de la pièce pour la télévision, le «tableau blanc avec des liserés blancs », œuvre des années 1970 d'un peintre fictif, Antrios ${ }^{51}$, autour duquel les trois amis vont discuter et se déchirer, n’arrive pas à trouver sa place dans l'appartement de Serge, son acquéreur ${ }^{52}$. La toile, dont on ne peut bien sûr, du point de vue du spectateur,

48. Voir Marie-Ève Marchand, «Le musée d'art et l'exposition: construction d'un discours », Dire, vol. 18, n² 2 , hiver 2009, p. 14-21.

49. Walter Moser, «L'interartialité: pour une archéologie de l'intermédialité », p. 78.

50. Yasmina Reza, Théâtre. L'homme du hasard. Conversations après un enterrement. La traversée de l'hiver. "Art», p. 193.

51. Ce peintre imaginaire a été inspiré par les artistes du courant minimaliste des années 1960 et 1970 qui ont expérimenté les formes «blanc-sur-blanc» en peinture, comme Martin Barré et Robert Ryman, inspirés eux-mêmes par certaines œuvres de Kasimir Malevitch, notamment par son Carré blanc sur fond blanc (1918).

52. Par exemple, p. 216: «Marc (désignant l'Antrios): Tu vas le mettre où?/Serge: Pas décidé encore [...]/Marc: Tu vas l'encadrer?/Serge (riant gentiment) : Non !... Non, non... [...] Ça ne s'encadre pas. [...] Volonté de l'artiste. Ca ne doit pas être arrêté. Il y a un entourage... » Ou encore: "Yvan (désignant l'Antrios): Tu vas le mettre où ?/Serge : Je ne sais pas encore./Yvan: Pourquoi tu ne le mets pas là?/Serge: Parce que là il est écrasé par la lumière du jour...» Yasmina Reza, Théâtre. L'homme du hasard. Conversations 
dire si elle est réellement peinte ou non, circule dans l'espace, apparaît et disparaît en fonction des humeurs des protagonistes, contrairement aux reproductions de «croûtes" montrées sur les murs de Marc et d'Yvan, qui ne sont clairement que décoratives. Au début de la pièce, le tableau est posé «à même le sol³ ", face au public, dans l'appartement de Serge, et Serge et Marc le contemplent. Marc va en discuter chez Yvan, qui promet à Marc qu'avec lui, Serge «rira ». Dans la scène entre Serge et Yvan qui a lieu dans l'appartement de Serge, le tableau n'est d'abord pas visible, mais Serge ne tarde pas à exhiber fièrement sa nouvelle acquisition ${ }^{54}$. Lors de la seconde confrontation entre Marc et Serge, toujours chez ce dernier, la toile est encore làns. La conversation s'envenime quand arrive Yvan, en retard au rendez-vous des trois amis. Sentant que sa présence ne fait qu'envenimer la situation, Serge sort le tableau de la pièce ${ }^{56}$. La tension croît toujours, Serge et Marc frôlent l'altercation, se retournent contre Yvan, qui craque. Serge revient finalement accrocher le tableau en fond de scène et tend un feutre à Marc, qui dessine dessus un bonhomme en skis ${ }^{57}$. La scène suivante voit le dénouement du mini-drame: Serge et Marc nettoient le tableau sous le regard approbateur d'Yvan, puis la lumière isole la toile à nouveau immaculée jusqu'à la fin de la pièce. Ces déplacements successifs rendent le tableau «vivant», créent un enjeu proprement théâtral, car la toile est dès lors reliée à la fois à l'intrigue, c'est-à-dire au temps, et à l'espace.

Lappartement de Serge présente un intérieur «monacal ${ }^{58}$ », qui fait écho à sa conception de la vie, pressée par les obligations privées et professionnelles. Il semble ainsi poussé à «aller à l'essentiel» - comme en peinture, lui fait ironiquement remarquer Marc, «où [il a] avantageusement éliminé forme et

après un enterrement. La traversée de l'hiver. «Art», p. 225. Notons également que le mot «art», dans le titre de la pièce, est entre guillemets, comme s'il était impropre, inadéquat (à l'amitié entre Marc, Serge et Yvan, et, peut-être, à la définition canonique de l'art).

53. Yasmina Reza, Théâtre. L'homme du hasard. Conversations après un enterrement. La traversée de l'hiver. «Art», p. 195.

54. Yasmina Reza, Théâtre. L'homme du hasard. Conversations après un enterrement. La traversée de l'hiver. «Art», p. 204-205.

55. Yasmina Reza, Théâtre. L'homme du hasard. Conversations après un enterrement. La traversée de l'hiver. «Art», p. 213.

56. Yasmina Reza, Théâtre. L'homme du hasard. Conversations après un enterrement. La traversée de l'hiver. «Art», p. 233.

57. Yasmina Reza, Théâtre. L'homme du hasard. Conversations après un enterrement. La traversée de l'hiver. «Art», p. 248-249.

58. Yasmina Reza, Théâtre. L'homme du hasard. Conversations après un enterrement. La traversée de l'hiver. «Art», p. 204. 
couleur, ces deux scories ${ }^{59}$ ». Cette remarque anodine rappelle que Yasmina Reza monte sa pièce pour la première fois en 1998, c'est-à-dire dans une période de débats sur la valeur ou l'authenticité de l'art contemporain en France ${ }^{60}$. Surtout, elle fait référence à l'obsession moderniste de pureté picturale, qui voulait justement éliminer tout ce qui est théâtral, pour atteindre, selon le terme greenbergien, la «planéité ». Michael Fried a reconduit, dans Art and Objecthood, l'argument de Greenberg, en montrant comment «l'objectité » de l'art « littéraliste » (minimal) reste de la sensibilité théâtrale, en s'attachant à l'inclusion du spectateur dans l'œuvre d'art, par sa situation envers l'objet, mais aussi par la présence «scénique » même de l'œuvre, la pression quasi agressive qu'elle exerce sur le spectateur et son exigence intrinsèque d'être «mise à distance » par lui pour être appréciée. L’approche littéraliste veut définir une nouvelle esthétique selon l'angle de vue, l'éclairage, le contexte spatial, la dimension anthropométrique et la disposition anthropocentrique de l'œuvre ${ }^{61}$. En fin de compte, mettre sous les yeux du spectateur de théâtre un tableau blanc serait une tautologie pour un minimaliste, alors que pour Fried, ce serait un non-sens total, car la "présenteté » de la peinture doit s'opposer à la durée théâtrale pour survivre. Dans «Art», la mise en scène retranscrit à la fois la dimension spectaculaire de l'art moderne, en dehors de l'espace muséal (inclusion du jugement du spectateur sur l'œuvre par son rire, mais aussi dans l'histoire qui a pour centre le tableau, rapport de distance physique et critique, présence évidente/disparition de l'œuvre sur scène, etc.), une grande partie de la dimension socio-médiale de la peinture (achat des collectionneurs, attitudes réfléchies des contemplateurs du tableau sur scène, gestes «caricaturés» du peintre en création ${ }^{62}$, etc.) et la mise en question de la valeur de l'art contemporain (question du prix, jugement esthétique

59. Yasmina Reza, Théâtre. L'homme du hasard. Conversations après un enterrement. La traversée de l'hiver. "Art», p. 213.

6o. Voir l'analyse de Nathalie Heinich, «L'art contemporain exposé aux rejets: contribution à une sociologie des valeurs", Hermès, n² 20, 1996, p. 193-204; pour les débats autour de la monochromie, voir Denys Riout, La peinture monochrome: histoire et archéologie d'un genre, Nîmes, Éditions Jacqueline Chambon, coll. « Rayon art», 1996, p. $105-118$.

61. Michael Fried, Contre la théâtralité: Du minimalisme à la photographie contemporaine, p. 120-125.

62. Quand il dessine sur la surface du tableau blanc, Marc imite au début les gestes caractéristiques du peintre (distance, mesure des proportions, etc.), puis finit son œuvre en quelques traits grossièrement esquissés. Yasmina Reza, Théâtre. L'homme du hasard. Conversations après un enterrement. La traversée de l'hiver. «Art», p. 249. 
ou «esthésique», d'après les registres de valeur définis par Nathalie Heinich ${ }^{63}$, herméneutique, etc.).

La toile blanche est une solution technique astucieuse pour éviter les dangers de la reproduction d'une œuvre d'art qui serait certainement coûteuse et de mauvaise qualité : le spectateur y projette ce qu'il veut, comme le font d'ailleurs les trois amis dans leurs discussions sur le contenu du tableau, des nuances, des couleurs, des lignes, «une pensée ${ }^{64}$, ou une «vibration ${ }^{65} »$. Voici par exemple la première description technique du tableau par Marc, le pragmatique, qui le présente à l'imagination de son ami Yvan:

Marc : Représente-toi une toile d'environ un mètre soixante sur un mètre vingt... fond blanc... entièrement blanc... en diagonale, de fines rayures transversales blanches... tu vois... et peut-être une ligne horizontale blanche en complément, vers le bas...

Yvan : Comment tu les vois? [ ...]

Marc: Parce que je les vois. Parce que mettons que les lignes soient légèrement grises, ou l'inverse, enfin il y a des nuances dans le blanc! Le blanc est plus ou moins blanc !66

Serge, quant à lui, se perd dans sa contemplation attentive :

Pour moi, il n'est pas blanc. [...] Il a un fond blanc, avec toute une peinture dans les gris... Il y a même du rouge. On peut dire qu'il est très pâle. Il serait blanc, il ne me plairait pas. Marc le voit blanc... C'est sa limite ${ }^{67} \ldots$

Yvan paraît plus hésitant à définir son goût, sommé qu'il est de le justifier face à son exigent ami :

63. Nathalie Heinich, «L'art contemporain exposé aux rejets: contribution à une sociologie des valeurs », p. 194.

64. Yasmina Reza, Théâtre. L'homme du hasard. Conversations après un enterrement. La traversée de l'hiver. "Art», p. 210.

65. Yasmina Reza, Théatre. L'homme du hasard. Conversations après un enterrement. La traversée de l'hiver. "Art», p. 206. De même, pour Robert Ryman par exemple, le tableau n'est pas un monochrome, il met l'accent sur d'autres choses et d'autres sens, il procure un «plaisir de voir». Denys Riout, La peinture monochrome. Histoire et archéologie d'un genre, p. 113-116.

66. Yasmina Reza, Théâtre. L'homme du hasard. Conversations après un enterrement. La traversée de l'hiver. «Art», p. 200-201.

67. Yasmina Reza, Théâtre. L'homme du hasard. Conversations après un enterrement. La traversée de l'hiver. «Art», p. 212. 
Marc (à Yvan): Et tu vois quoi comme couleur?...

Yvan: Je vois des couleurs... Je vois du jaune, du gris, des lignes un peu ocre...

Marc: Et tu es ému par ces couleurs. [...] La vérité? Ces couleurs te touchent?

Yvan: Oui, ces couleurs me touchent. [...]

Marc: Il n'y a pas de couleurs. Tu ne les vois pas. Et elles ne te touchent pas.

Yvan : Parle pour toi ${ }^{68}$ !

Ces jugements esthésiques sont également des jugements de personnes qui incriminent l'étroitesse d'esprit, l'incompréhension, ou le manque de culture de l'une ou de l'autre et font basculer le registre artistique dans le registre théâtral. Évidemment, le spectateur ne peut s'approcher de la toile, il doit se fier à ce qu'en disent les personnages, se laisser convaincre par la fiction du tableau. Il ne peut «voir quelque chose» qu'à la fin de la pièce, où Marc, dans une ultime provocation, rend la toile figurative, et y projette matériellement, grâce au feutre d'Yvan, son humour, sous les traits d'un bonhomme en skis sur une piste enneigée, selon le tracé hypothétique d'une des fameuses «rayures transversales blanches ».

Le spectateur se voit donc en définitive imposer l'interprétation du personnage dominant du trio, et qui se définit fièrement comme tel ${ }^{69}$, non pas en tant que critique d'art ou amateur éclairé, mais bien au contraire comme seul homme rationnel et indépendant des modes du siècle, avant que la toile ne retrouve une neutralité réconciliatrice. L'insistance sur la blancheur du tableau, couleur la plus intense, qui absorbe tout le spectre, accentuée par le dépouillement du décor, permet d'en faire une métaphore de l'objet théâtral, car le propre de la scène est d'être «le seul terrain de confrontation authentique entre les différents types d'images qui nous entourent. Le théâtre, qui par nature est un lieu d'échange et d'interaction entre tous les arts convoqués [...] constitue le champ idéal où toutes nos images, protagonistes à la fois du monde réel et du

68. Yasmina Reza, Théâtre. L'homme du hasard. Conversations après un enterrement. La traversée de l'hiver. «Art», p. 226-227.

69. Marc à Serge: «Je t'ai toujours su gré de me considérer comme à part. J'ai même cru que cet à part était de l'ordre du supérieur...» Yasmina Reza, Théâtre. L’homme du hasard. Conversations après un enterrement. La traversée de l'hiver. "Art», p. 241. 
théâtre, peuvent dialoguer entre elles et avec nous, être mises en crise ${ }^{70}$. " Béatrice Picon-Vallin souligne que la critique de l'image est souvent implicite dans sa « convocation scénique » : le déplacement de l'image télévisuelle hors de son flux contextuel à la scène crée une distance nécessaire pour désigner le leurre ou le scandale, par exemple. De même, la présence d'une peinture au théâtre interroge ici son contenu, mais plus largement sa raison d'être hors du musée ou de la galerie, chez un particulier ou sur une scène. Dans «Art», ce questionnement intervient cependant sans perturber la relation spectateur-peinture habituelle, du moment qu'elle est placée face à lui, pleinement disponible à son regard et à sa critique; seul l'espace environnant le contact de l'œuvre, l'institution, diffère. Cette approche théâtrale de la toile est comparable à celle de Jarman de l'œuvre du Caravage, à ceci près que l'œil de la caméra balaie plus étroitement le tableau; la distance de l'œil à la toile est abolie par la technique cinématographique, elle permet un abord plus physique, de «corps à corps» avec la peinture, que la distance à la scène ne connaît pas. L'approche théâtrale reste ici abstraite, au niveau de la pensée. Il en va tout autrement dans le deuxième cas étudié.

Seuls est l'histoire d'un étudiant d'origine libanaise, Harwan, qui prépare une thèse sur «le cadre comme espace identitaire dans les solos de Robert Lepage ${ }^{71}$ ». Alors qu'il s'apprête à partir pour Saint-Pétersbourg rejoindre le metteur en scène, son père tombe dans le coma. Par un « dialogue à une voix » avec ce père absent, il renoue avec son passé, sa vie oubliée au Liban, et sa vocation pour la peinture qu’il a refoulée à cause de son exil en France puis au Québec. Mais, au fur et à mesure, on comprend que ce n'est pas le père qui a eu un accident cérébrovasculaire, mais Harwan lui-même, alors qu'il prenait des photos pour son passeport. Prisonnier de sa chambre d'hôpital et de sa conscience, il retrouve peu à peu les gestes de son enfance, quand il peignait les étoiles dans le ciel, les couleurs, le plaisir de peindre qu'il avait enfoui en lui. L'intrigue, semi-autobiographique, se concentre autour d'un tableau de Rembrandt, Le retour du fils prodigue $e^{72}$, tiré de la parabole de l'Évangile selon saint Luc ${ }^{73}$, et thème central de la future création de Robert Lepage. Seul le côté gauche du tableau, qui représente un père, de

70. Béatrice Picon-Vallin, «Hybridation spatiale, registres de présence », p. 33.

71. Wajdi Mouawad, Seuls: chemin, texte et peintures, p. 132.

72. Huile sur toile - 262 x 206, 1669, musée de l'Ermitage, Saint-Pétersbourg.

73. Luc, 15, 11-32. Voir Évangiles. Lettres de Jean. Actes des Apôtres, d'après la nouvelle traduction des éditions Bayard, Paris, Gallimard, 2001, p. 242-243. Un père a deux fils. L'un part dissiper sa part d'héritage, et revient, ruiné, demander le pardon à son père pour ses péchés. Le père l'accueille par une fête, et ne lui demande aucun compte: il le croyait mort, et le voici revenu. 
face, posant les mains sur les épaules de son fils qui nous tourne le dos, est projeté à plusieurs reprises au fil du spectacle sur le mur-écran de l'espace de Harwan, tantôt en entier, tantôt en grossissant la seule figure du père, etc. La projection de fragments épars du tableau qui rythme la pièce déclenchera la catharsis finale et la réalisation de sa vocation par Harwan. Ici encore, le tableau est une métaphore, celle du désir de peindre chez le personnage principal. Il explique d'ailleurs ce que signifie pour lui ce tableau, dans la conclusion de sa thèse, inopinément retrouvée parmi ses affaires pendant sa longue période d'hospitalisation:

Et si, moi, je devais retourner vers ce qui m'attend, comment ferais-je pour le trouver, pour m'en souvenir? Qu'est-ce qui, depuis si longtemps espère mon retour? Qu'est-ce qui, s'il me voyait au bout de la route, en serait à ce point ému? Qu'ai-je donc quitté sans même le comprendre? Ai-je perdu toute mémoire? Comment diton mémoire en arabe ${ }^{74}$ ?

La projection sur le mur de scène du tableau rend sa présence fantomatique, suggestive, discrète et pourtant écrasante. Le clair-obscur des œuvres de Rembrandt accentue cette impression suggestive ainsi que l'aura du tableau. Mais pour atteindre cet effet, la remédiation de la peinture passe encore par l'écran, c'est-à-dire par un autre cadre, en plus de celui de l'ouverture scénique, qui redéfinit celui, initial, de l'œuvre originale de l'Ermitage, par l'œil de la caméra, ou plutôt par le contour de l'écran, qui oriente le regard et affecte le jugement du spectateur sur l'œuvre. C'est pour la clarté de l'histoire, donc pour des raisons qui relèvent purement de l'exigence diégétique de l'intrigue théâtrale, qu'un seul détail, un sens particulier, sont accentués, laissant dans l'ombre les autres personnages, la sœur, le frère, les serviteurs ou invités.

Grâce à l'intrusion de la projection vidéo, le théâtre peut intégrer l'œuvre de façon presque directe sur une scène, sans bien sûr retrouver la proximité de la salle d'exposition, pour le spectateur du moins. L'acteur, en revanche, peut avoir un contact corporel avec la peinture, celle qui est projetée sur le mur et le rideau mobile de la fenêtre et celle qu'il déverse des pots et des tubes qu'il trouve dans sa valise de voyage sur les feuilles blanches de sa thèse, puis sur des panneaux de plastique transparent qu'il déploie à la fin du spectacle. Dans son « odyssée » vers la peinture, il se couvre le corps de couleurs (rouge, vert, bleu, jaune, blanc) ${ }^{75}$, en badigeonne ses feuilles, applique sur les murs déployés comme un atelier d'artiste l'empreinte de son corps et de ses mains, recrée sa silhouette en agrafant

74. Wajdi Mouawad, Seuls: chemin, texte et peintures, p. 18.

75. Lors de son «bain», il se lave le corps avec de la peinture rouge. Wajdi Mouawad, Seuls: chemin, texte et peintures, p. 164-168. 
son pantalon, puis il se couvre le visage d'un masque au moment où, dans la vie consciente, les docteurs apprennent à sa famille qu'il peut revenir à lui mais qu'il aura perdu la vue ${ }^{76}$.

Par la magie de la projection, Harwan peut même se confondre avec le fils à genoux devant son père, sur le tableau de Rembrandt, avant de crever le papier où est projetée la toile pour apparaître à la place du visage du Père ${ }^{77}$, «dans le ventre du tableau », «à jamais dans son cadre ${ }^{78}$ ». Ces mouvements de va-et-vient entre l'acteur et la toile ne sont pas sans rappeler les allées et venues de la caméra de Godard au sein des tableaux reconstitués de Goya ${ }^{79}$, qui pourfendent la bi-dimensionnalité et la médialité de la peinture et permettent un véritable effet de dramatisation de l'œuvre vue différemment. Relief et vie apparaissent quand le corps entre en contact avec la matérialité du média, et délivre non seulement un autre sens, un sens personnel nouveau en lien avec la fable de Harwan, à travers un parcours presque psychanalytique, du fils au père, qui fait remonter le personnage aux origines de sa passion pour l'art, mais aussi une dimension esthétique inédite entre une œuvre et un homme, une perception sensuelle de la matérialité de la peinture réservée en principe au seul artiste, mais ici ouverte à tous les spectateurs par le truchement du corps de l'acteur. Cette expérience, très forte pour le spectateur, fait lointainement écho à un courant de peinture spectaculaire. Les Anthropométries d'Yves Klein remplaçaient aussi le pinceau par le modèle (des corps de femme). Ces performances picturales faisaient l'objet d'un cérémonial très réglé et fort médiatisé où le "grand prêtre », l'artiste en personne, ne se confrontait plus aux pigments, mais dirigeait son instrument à distance, selon sa volonté ou son inspiration. S'il ne s'agit certainement pas d'une citation de ce travail de la part de Mouawad, celui-ci retrouve la dimension rituelle de l'exercice; mais alors que les Anthropométries laissent une impression de distance un peu glacée, où l'œil mi-sérieux mi-narquois de l'artiste se

76. Dans «Buste », il verse de la peinture blanche sur son masque, puis de la peinture rouge coule de la fente de ses yeux. Wajdi Mouawad, Seuls: chemin, texte et peintures, didascalie p. 169 et 171; dans «Nettoyage ", il s'enduit de blanc et repeint la silhouette sur le mur en blanc. Wajdi Mouawad, Seuls: chemin, texte et peintures, didascalie p. 174; enfin, il peint « longtemps » dans son « atelier» («Les gestes du peintre», Wajdi Mouawad, Seuls: chemin, texte et peintures, didascalie p. 177).

77. Dans les dernières sections de la pièce intitulées «L'esprit du peintre» et «Le lieu du peintre ", Wajdi Mouawad, Seuls: chemin, texte et peintures, didascalie p. 182-185, dans l'édition du texte.

78. Wajdi Mouawad, Seuls: chemin, texte et peintures, didascalie p. 184.

79. Walter Moser, «L'interartialité: pour une archéologie de l’intermédialité », p. 89. 
pose tel un démiurge sur sa création, poussant la captation du spectacle aux limites du voyeurisme, l'expérience à laquelle l'acteur et metteur en scène convie son spectateur est pleinement participative, malgré, et même à cause, de sa distance. L'implication de l'acteur et «créateur» - c'est, il semble, la volonté de Mouawad que de créer une « œuvre originale » sur scène, bien qu'un critique d'art puisse s'interroger sur le statut de cette œuvre - est dans ce cas essentielle; loin de refuser le contact charnel avec la peinture dans toute sa médialité, il nous confond dans notre statut d'observateurs respectables et éloignés, nous oblige à nous laisser imbiber par la peinture, à crever le cadre avec le héros de cette histoire, comme Harwan nous a déjà entraînés à la poursuite de Lepage en Russie ou, entre Montréal et le Liban, au chevet de son père malade.

Ainsi, c'est moins une question d'esthétique picturale que d'esthétique théâtrale que me semble proposer Seuls. Peu importe au fond que Mouawad réalise sur scène une œuvre d'art authentique. Le spectateur n'est pas appelé à en juger, comme c'est le cas dans «Art». En revanche, il est invité à une expérience picturale rare au cours d'une représentation théâtrale somme toute classique, et à travers une esthétique proprement théâtrale, qui lui fait affronter durant tout le spectacle le problème de la distance et de l'illusion de la peinture. Les effets vidéo déjà décrits et la construction de l'intrigue elle-même s'ébauchent, me semblet-il, à partir de ce jeu de la distance et de l'illusion. Harwan court à travers le monde pour mieux s'en couper, les seuls contacts qu'il entretient avec sa famille sont téléphoniques, il revit son ancien amour sur un écran d'ordinateur ; le travail lui sert de prétexte à l'isolement, alors que concrètement sa thèse n'avance pas, ou pas comme il voudrait. À vrai dire, il ne sait pas ce qu'il cherche, ou mieux, par quoi il sera trouvé. Dans cette quête vitale, il se remémore un passé perdu, à l'aide de bribes sonores (voix de son père et de sa sœur, chants d'oiseaux, souffle du vent, aboiements qui évoquent le Liban), de paroles oubliées, d’indices ténus charriés par ses sens attentifs, mais quelle réalité ont-ils vraiment? Alors qu'il croyait assister au coma de son père, c'est lui-même qui se trouve soudain confronté à la mort. Du moment que la réalité de sa situation lui apparaît, il prend le problème à bras-le-corps, pour ainsi dire, pour briser l'illusion qu'il avait sur ses vrais désirs, combler la distance qui le séparait des autres et de lui-même.

Wajdi Mouawad a ici trouvé une voie très originale pour présenter la peinture sur scène, à travers le corps de l'acteur, le principal «média» du théâtre, et surtout les émotions et les souvenirs qu'elle suscite, visuels et auditifs, à travers projection vidéo et performance artistique live, proche des Action Paintings de Jackson Pollock ou des Anthropométries de Klein, sons de voix, bruits, etc., qui s'intègrent au média et à l'histoire et nous font partager sensations et réflexions. 
Hors du musée, point de salut, semble-t-il. Sur scène, le tableau ne peut être qu'une métaphore de l'art, pas une œuvre authentique. Les deux pièces portent toutefois des œuvres d'art sur la scène en les théâtralisant, en les inscrivant dans une narration et un décor où elles deviennent en quelque sorte des protagonistes, qui peuvent dès lors dialoguer, interagir avec les acteurs en présence. C'est ainsi qu'elles font perdre leur «cadre » ${ }^{80}$ et leur «opacité» à la toile, bien qu'elles aient mis en scène, par des procédés qui rejoignent souvent ceux du cinéma et surtout qui utilisent l'écran comme nouveau cadre, la médialité de la peinture, tel que le dit Walter Moser. "Art» et Seuls s'achèvent d'ailleurs toutes deux sur le mot «opacité », pour désigner soit l'impossibilité de justifier quelque interprétation d'une œuvre d'art, soit le champ illimité des possibles sens et sensations qu'elle éveille, ce qui revient somme toute au même. À la fin de «Art», Marc, seul devant le tableau blanc, produit une interprétation différente de celle qu'il a émise au début de la pièce:

Sous les nuages blancs, la neige tombe. On ne voit ni les nuages blancs, ni la neige. $\mathrm{Ni}$ la froideur et l'éclat blanc du sol./Un homme seul, à skis, glisse./La neige tombe./ Tombe jusqu'à ce que l'homme disparaisse et retrouve son opacité $e^{81}$.

Dans Seuls, la conclusion «magistrale » de la thèse de Harwan, que son professeur lit, éclaire le sens du cadre-écran de projection dans les solos de Robert Lepage, «lieu de tous les possibles », où les personnages échappent «aux lois du temps et de l'espace et à la loi de la gravité»:

Il est donc de nature paradoxale: le lieu fini est celui de l'infini, la limite offre l'illimité, la frontière l'ouverture, la borne l'insoupçonné. L'opacité disparaît et la surface, sur laquelle le regard s'arrêtait, révèle une profondeur où l'esprit n'étouffe pas sur lui-même mais s'ouvre sur un espace où le corps, enfin libéré, aborde le rivage des sensations retrouvées ${ }^{82} \ldots$

Alors que les exemples pris par Moser présentent une interartialité qui révèle tour à tour la médialité de chaque art considéré, peinture et cinéma, au théâtre aucun média ne semble l'emporter sur l'autre. Au-delà même d'une révélation de

8o. Dans «Art» le tableau de Serge «ne s'encadre pas», car il est entouré d'une «sorte de craft... [c]onfectionné par l'artiste», qui laisse le tableau proprement sans limites. Yasmina Reza, Théâtre. L'homme du hasard. Conversations après un enterrement. La traversée de l'hiver. «Art», p. 216. Dans Seuls, le personnage crève la limite de la toile, va et vient dans le tableau et dans la peinture.

81. Yasmina Reza, Théâtre. L'homme du hasard. Conversations après un enterrement. La traversée de l'hiver. «Art», p. 251.

82. Wajdi Mouawad, Seuls: chemin, texte et peintures, didascalie p. 182. 
la médialité d'un art par l'autre, telle que «Art» la met en scène en s'amusant de la médialité de la peinture, Seuls offre la possibilité de réfléchir sur l'esthétique théâtrale grâce aux médialités conjuguées de la peinture et du théâtre. La pièce permet en effet au spectateur d'aller plus loin dans les niveaux de perception. Deux raisons peuvent expliquer une telle situation: le recours, régulier et productif chez Mouawad (et Lepage), à l'écran vidéo pour remédier la peinture, qui décale d'un degré la relation directe théâtre-peinture; la spécificité médiatique propre au théâtre depuis son apparition, qui passe par un texte, un dialogue, au minimum une intrigue qui va d'un point $\mathrm{A}$ à un point $\mathrm{B}$. Le passage par un troisième média ou art (vidéo ou écriture) est-il constitutif de l'interartialité au théâtre? Cette question nous amène à penser la définition de la médialité du théâtre et de la théâtralité.

\section{III- CONCLUSION : POUR UNE AUTRE INTERARTIALITÉ ? LA MISE EN QUESTION

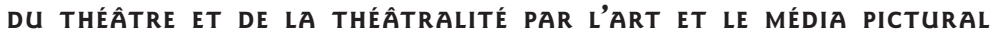

Béatrice Picon-Vallin, dans son article «Hybridation spatiale, registres de présence $^{83}$ », démontre que la présence de l'écran implique une nouvelle définition de la scène et de la mise en scène, qui utilise précisément le vocabulaire de la peinture. La «bidimensionnalité » du théâtre de Robert Wilson, par exemple, «est une réponse à la manière de percevoir du public que toutes les technologies de l'image ont contribué à façonner dans son quotidien. Wilson dématérialise le plateau, peignant ou dessinant avec la lumière ou la couleur. Sans utiliser directement des images, il déplace leur effet sur le plateau ${ }^{84}$. » Grâce aux effets vidéo et aux technologies de pointe, Wilson recrée des images virtuelles, qui évoquent les arts qui y sont liés, la peinture, le cinéma, ou le numérique. La matérialité du plateau de théâtre est brisée par l'intégration de toutes sortes d'images fixes ou animées, peintes ou projetées, en direct ou non, qui empruntent la «technicalité » d'un autre art pour leur production (pinceau, crayons, couleur, lumière), déplaçant ainsi l'effet de l'image sur la scène pour créer un sentiment de reconnaissance chez le spectateur ${ }^{85}$. Toute surface plane devient un écran: «Pour Piscator déjà, l'écran en scène ne différait pas de la surface plane des

83. Béatrice Picon-Vallin, «Hybridation spatiale, registres de présence».

84. Béatrice Picon-Vallin, «Hybridation spatiale, registres de présence », p. 19 (nous soulignons).

85. Est-ce à dire que les codes théâtraux sont tombés en désuétude et ne sont plus reconnus par les spectateurs de théâtre eux-mêmes? 
toiles prêtes à être peintes ${ }^{86}$. » Avec la photo projetée, l'image cinématographique, la vidéo, les allers et retours hors du cadre scénique sont possibles sans quitter la scène, car ces technologies, utilisées simultanément dans leur diversité, abolissent la frontalité «même dans le cas d'un spectacle frontal », du moment que l'image «dote le spectateur de plusieurs regards. » ${ }^{87}$ L'effet produit s'apparente au "cubisme », par la combinaison du détail et du plan général, du gros plan qui dématérialise un corps et de sa silhouette entière, du plan de profil et d'un acteur de face...

Les possibilités de stratification, de surimpression intensifient encore la coexistence d'espaces et de temps hétérogènes sur la scène, dans un processus d'hybridation où s'approfondit l' «entre-deux» caractéristique de l'espace théâtral ${ }^{88}$.

L'espace théâtral se situe entre deux «espaces», celui de l'acteur et du spectateur, du texte (ou du son) et du sens, de l'image et de sa réception, mais il s'immisce aussi entre un média et un autre média, ou même un art et un autre art, au cœur de la relation entre les deux, entre distance et illusion. Art du mouvement, du dialogue, il fait dialoguer les arts. La résistance du média ou de l'art intégré sur scène souligne de façon tout à fait nette cette caractéristique de l'espace théâtral, et l'exemple de la peinture constitue, comme on l'a vu, un cas approprié, que ce soit au théâtre ou au cinéma, ainsi que l'analyse du film de Godard par Walter Moser le montre ${ }^{89}$. Le récit fonctionne comme une «exigence extérieure » à la peinture, mais intérieure au cinéma. Aussi Godard filme-t-il l'impossibilité de faire un film à partir de peinture: dans un film qui se propose de reconstituer une ébauche d'histoire de l'art, on ne peut parler que de cinéma, semble vouloir dire le réalisateur. Non pas que la tentative du film, reconstituer des œuvres d'art en scènes vivantes, ne soit pas intéressante en soi; mais les enjeux esthétiques sont différents. Ce sont les débats entre le cinéaste et les personnages ou le producteur, relevés par Moser, sur la façon de faire du cinéma sur la peinture qui empêchent le film de se réaliser, tout comme les discussions animées entre Marc, Serge et Yvan à propos de l'Antrios créent une brouille entre les amis, moment de «mise en crise» dans les deux cas que

86. Béatrice Picon-Vallin, «Hybridation spatiale, registres de présence », p. 25.

87. La multiplicité des dessins, des montages de dessins, ou la superposition des couleurs sur une «toile», comme la séquence «Corps» de Seuls en fait voir, contribue à cette pluridirectionnalité du regard.

88. Béatrice Picon-Vallin, «Hybridation spatiale, registres de présence», p. 27.

89. Walter Moser, «L'interartialité: pour une archéologie de l'intermédialité », p. $87-91$. 
l'intrigue cherche à résoudre. Nous avons parlé plus haut du choix de Godard pour une esthétique du fragment, de l'inachevé (découpage en portraits des protagonistes lors de la reconstitution des tableaux de Goya, mélange des personnages des différentes scènes avec le personnel technique qui donne l'impression d'un "chaos créateur ${ }^{90}$ ", etc.) qui métaphorise le travail du peintre en action. La pièce de théâtre aussi, dans un certain sens, reste inachevée: dans "Art», les amis repartent à zéro et envisagent une «période d'essai91 », Marc «tente» une nouvelle interprétation du tableau blanc; toute l'histoire de leur amitié reste cependant à reconstruire. Les esthétiques diffèrent parce que les enjeux des deux arts sont eux-mêmes différents, à cause du fonctionnement propre au média utilisé. Le message politique du tableau de Goya change du moment qu'on pénètre dans le tableau et qu'on voit les oppresseurs de face grâce au mouvement de caméra qui rompt la matérialité et la bidimensionnalité du tableau ${ }^{92}$. Dans le cas du théâtre, la complexité médiatique et les messages qu’elle véhicule nuance encore plus la situation. L'interprétation objective, ou du moins dépassionnée de l'Antrios, qui apparaît à la première scène dans toute sa pureté significative en quelque sorte, offerte au regard de tous, est perturbée dès lors que deux des personnages, liés par une vieille histoire d'amitié, s'opposent dans leur conception esthétique qui se traduit en un discours admiratif pour l'un, péjoratif et ironique pour l'autre. On le voit, l'intrigue d'une part, le texte d'autre part, mais aussi le jeu des acteurs et leur attitude face au tableau, bref la performativité, sont autant de paramètres à prendre en compte dans le jeu de l'interartialité.

Espace hybride, espace du média, la scène peut présenter toutes les contradictions, tous les médias et tous les arts dans leur problématique et leur esthétique intrinsèque. C'est pourquoi Godard nous semble celui des deux exemples proposés par Moser qui indique le plus le sens d'une véritable interartialité, c'est-à-dire d'une relation entre les arts plus qu'entre les médias, qu'indique aussi l'analyse de l'interartialité au théâtre. Dans son film, tous les aspects esthétiques de la peinture (composition, éclairage, fragmentation, mais aussi métaphore de l'œuvre inachevée, débat sur l'art de peindre et de «cadrer» le film) créent le média, son fonctionnement, son discours et son «sémantisme ", qui va permettre de rendre compte de son objet, dans un fructueux dialogue entre les arts, tandis que Jarman utilisait seulement le média cinéma pour retranscrire une autre

90. Walter Moser, «L'interartialité: pour une archéologie de l'intermédialité», p. 88. 91. Yasmina Reza, Théâtre. L'homme du hasard. Conversations après un enterrement. La traversée de l'hiver. «Art», p. 250.

92. Walter Moser, «L'interartialité: pour une archéologie de l'intermédialité», p. 89. 
médialité et une histoire, en gardant l'une de ses caractéristiques fondamentales, le déroulement linéaire et accompli du temps, qui présuppose un début et une fin. Prendre exemple sur Godard oblige ainsi à repenser ce qu'est l'esthétique du théâtre et sa médialité propre, sa théâtralité. Une autre manière de poser la question serait peut-être: quelle esthétique crée quel média?

Walter Moser l'a bien suggéré: le dialogue interartiel, en faisant saillir l'opacité médiatique d'un art, offre un champ d'analyse dont nous avons déjà exploré certaines pistes dans le point précédent. Le discours autour du tableau de «Art», par exemple, point focal de la narration, qui dit son histoire, son prix, les réactions qu'il suscite chez les personnages, comment il intervient dans leur vie, etc., par l'entremise du dialogue, recrée le discours critique polyphonique, pour ne pas dire cacophonique, du «Gotha des grands amateurs d'art» comme dit $\mathrm{Marc}^{93}$, mais en le banalisant, en le rendant familier, intime, pour les besoins de la comédie. Il reprend donc plusieurs aspects sociomédiaux de la peinture les plus adaptés à sa propre médialité grâce à un média commun, le langage, pour le porter sur la scène; en même temps, les contraintes du genre boulevardier, voire le type de public susceptible de venir voir une pièce intitulée "Art», qu’on peut supposer cultivé et amateur de bons mots, définissent des préoccupations et un ton certes légers, mais non dépourvus d'une certaine profondeur, qui écartent ce discours critique d'une théorie propre aux connaisseurs et spécialistes.

Seuls rappelle beaucoup la démarche godardienne dans sa recherche esthétique : le découpage du tableau en gros plans projetés sur écran, le montage de plans filmés des dessins de Harwan, de ses photos personnelles, d'images hétéroclites, mis en parallèle avec les empreintes corporelles de l'acteur sur les murs, invitent à voir la peinture autrement qu'elle ne peut l'être au musée, recadrée dans des limites différentes, qui font porter sur elle un autre regard, pluriel, celui de l'intermédialité, qui dépasse et transforme celui du spectateur. Le passage par la vidéo redéfinit les sens de la peinture, du théâtre et de l'écran lui-même. Par un autre média et les techniques de l'image, du gros plan, du montage et du son, Mouawad arrive à créer une autre esthétique de la peinture, qui n'est pas du théâtre pur, ni de la vidéo pure, mais un média hybride, qui serait, peut-être, le propre de la théâtralité. L'acte de peindre lui-même, montré et décuplé par les gestes, les cris, le corps de l'acteur, qui peint sur de grands panneaux avec rage et délectation, transite par un autre média, qui, comme l'écran, reçoit et transmet des messages qui le façonnent (Harwan entend des

93. Yasmina Reza, Théâtre. L'homme du hasard. Conversations après un enterrement. La traversée de l'hiver. «Art», p. 203. 
sons, des paroles issus d'un autre monde, du monde conscient où vivent son père et sa sœur ou de celui du Liban oublié). Le corps et chacun de ses membres (pieds, mains, buste, tête...) modèlent en retour la matérialité de la peinture, devenant tour à tour pinceaux, surface enduite de peinture, écran de projection du tableau.

La théâtralité n’apparaît donc évidemment pas avec l'avènement de la vidéo sur scène, mais bien avec le média. En renversant la proposition de Moser, on pourrait dire que si l'interartialité fait découvrir la médialité de l'art, le média fait apparaître son artialité, du moins celle du théâtre. 